\title{
Moving-edge detection via heat flow analogy
}

\author{
Cem Direkoğlu*, Mark S. Nixon \\ School of Electronics and Computer Science, University of Southampton, UK
}

\section{A R T I C L E I N F O}

\section{Article history:}

Received 21 September 2009

Available online 18 September 2010

Communicated by T.K. Ho

\section{Keywords:}

Moving-edges

Feature extraction

Image processing

Computer vision

Heat flow

\begin{abstract}
A B S T R A C T
In this paper, a new and automatic moving-edge detection algorithm is proposed, based on using the heat flow analogy. This algorithm starts with anisotropic heat diffusion in the spatial domain, to remove noise and sharpen region boundaries for the purpose of obtaining high quality edge data. Then, isotropic and linear heat diffusion is applied in the temporal domain to calculate the total amount of heat flow. The moving-edges are represented as the total amount of heat flow out from the reference frame. The overall process is completed by non-maxima suppression and hysteresis thresholding to obtain binary movingedges. Evaluation, on a variety of data, indicates that this approach can handle noise in the temporal domain because of the averaging inherent of isotropic heat flow. Results also show that this technique can detect moving-edges in image sequences, without background image subtraction.
\end{abstract}

(c) 2010 Elsevier B.V. All rights reserved.

\section{Introduction}

Segmenting moving objects is a challenging and important task in computer vision. It has many applications such as surveillance, video communication, traffic monitoring, people tracking, content-based image coding and image compression. There are many moving object segmentation techniques (Kim and Hwang, 2002; Ahn et al., 2004; Myerscough and Nixon, 2004; Kim, 2005; Zhang and Zhao, 2006; Dewan et al., 2007; Hwang and Clark, 1990a,b), which are based on moving-edge detection. We first review some of these techniques, and then describe the use of the heat flow analogy in image analysis which also includes the proposed model and its advantages as a feature extraction.

\subsection{Related works}

Moving-edge detection has attracted attention for moving object segmentation. Kim and Hwang (2002) segment moving objects based on extracted moving-edges. Moving-edge points are generated based on processing the frames difference edge map, current frame edge map, and background edge map. These spatial domain edge maps are obtained using the Canny edge detector, which involves Gaussian convolution to suppress noise. Ahn et al. (2004) introduced a moving-edge detection algorithm to implement a home security system. Their algorithm extracts edge segments from the current image and eliminates the background edge seg-

\footnotetext{
* Corresponding author. Address: School of Electronics and Computer Science, University of Southampton, SO17 1BJ, UK. Tel.: +44 (0)7785990782; fax: +44 (0)2380594498.

E-mail address: cdirekoglu@googlemail.com (C. Direkoğlu).
}

ments by matching them with a reference edge list, where the reference edge list is generated from the set of training background images. Myerscough and Nixon (2004) developed a moving-edge detector by extracting feature points and feature velocities from a sequence of images based on using phase congruency. The benefits of using phase congruency are its illumination invariance and good localization. In addition, since phase congruency is illumination invariant, there is no need for threshold selection in edge detection. Kim (2005) proposed a moving-edge detection algorithm by using entropy and cross-entropy approaches. They calculate entropy in the current frame to determine spatial edges. Then, they apply cross-entropy between current and previous images to measure the dissimilarity. Finally, entropy and cross-entropy values are compared and thresholded to detect moving-edges. Zhang and Zhao (2006) proposed a moving-edge detection algorithm using wavelets to overcome noise. First, they find change detection mask in the wavelet domain. Then they apply Canny edge detection on the change detection mask to obtain the difference edge map. Finally, the difference edge map is compared with current frame edge map, background edge map and previous frame's moving-edges to obtain the current frame's moving-edges. Recently, Dewan et al. (2007) introduced an algorithm, which utilizes the three most recent consecutive frames to isolate moving-edges for moving object detection. They first calculate two difference images using those three consecutive frames. Then, the Canny edge detection algorithm is applied to generate difference edge maps. In the difference edge maps, edge pixels are grouped together to represent edge segments. Finally, since the moving-edges of the middle frame are common in both difference edge maps, they apply matching algorithm by using the size, shape and position features of the edge segments. 


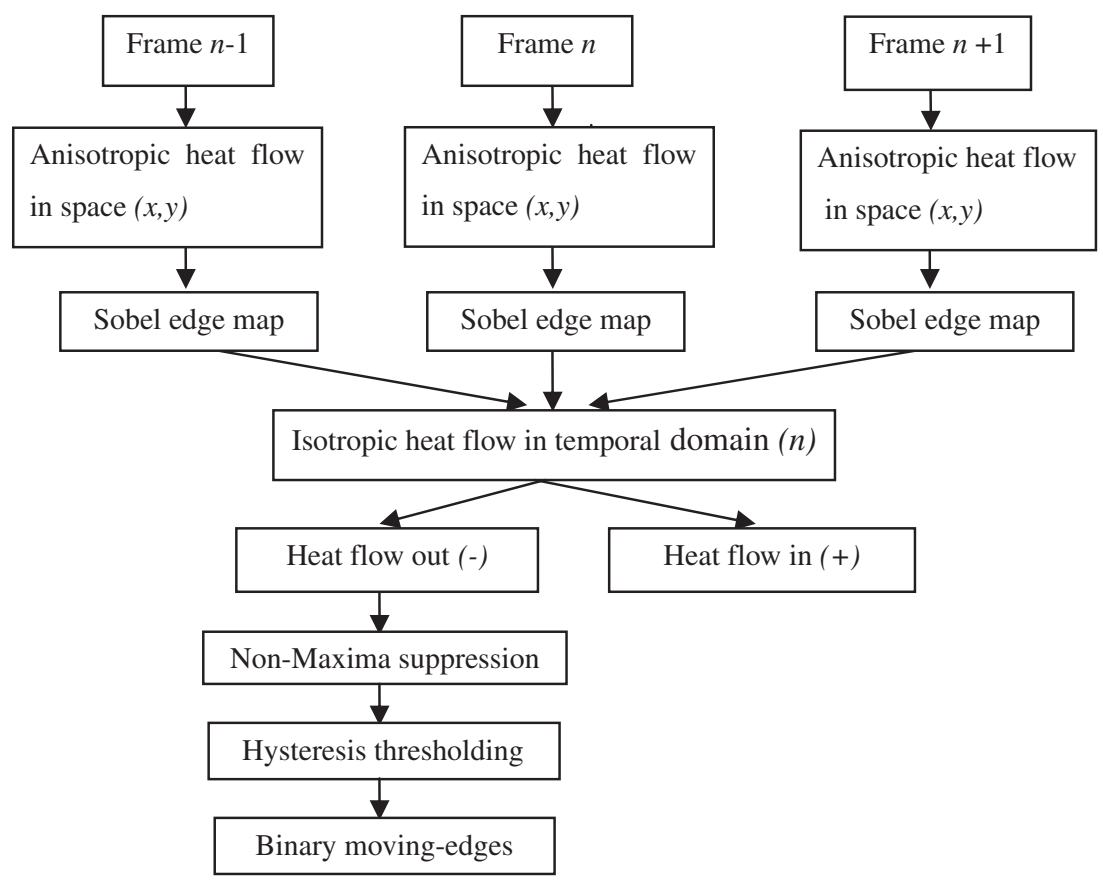

Fig. 1. Moving-edge detection.

\subsection{Using heat flow in image analysis}

The heat flow analogy has been deployed in various ways in image processing and computer vision. It has been used for: image smoothing and enhancement; region-based image segmentation; thinning; active contours; and motion analysis. Anisotropic diffusion, which was introduced to computer vision by Perona and Malik (1990), is a state-of-art image enhancement technique. In (Manay and Yezzi, 2003), the anti-geometric heat flow model was introduced for the segmentation of regions, where the flow was represented as diffusion through the normal direction of edges. A smooth shape extraction algorithm was also presented in (Direkoğlu and Nixon, 2007) by solving the particular heat conduction problems in the image domain. In (Ji and Feng, 2004), a new thinning algorithm was introduced based on time-reversed isotropic heat flow. A geometric active contour (Caselles et al., 1993; Malladi et al., 1995) was based on a curve moving in normal direction with its curvature dependent on speed, which is also called level set method (Osher and Sethian, 1988). The curve movement in the level set approach has also been achieved with geometric heat flow (Kimia and Siddiqi, 1994). In motion analysis, Makrogiannis and Bourbakis (2004) proposed a spatio-temporal anisotropic heat diffusion for motion activity measurement.

In our research, a moving-edge detection algorithm is proposed based on the heat flow analogy. This algorithm starts with anisotropic heat diffusion in the spatial domain to remove noise and sharpen region boundaries for the purpose of obtaining high quality edge maps. Once the enhanced edge maps are observed in three or more consecutive frames, isotropic and linear heat diffusion is applied in the temporal domain to calculate the total amount of heat flow. The moving-edge map is represented as the total amount of heat flow out from the reference frames. The overall process is completed by non-maxima suppression for thinning and then hysteresis thresholding to obtain binary moving-edges. Evaluation results indicate that this approach has advantages in handling noise in the temporal domain because of the averaging inherent in isotropic heat flow. Results also show that this technique can detect moving-edges in image sequences without using the background image. Note that the preliminary version and the summary of this work were presented in (Direkoğlu and Nixon, 2006; Nixon et al., 2009), respectively. In this paper, we extend the basis and evaluation of the new technique. Fig. 1 shows the proposed algorithm to obtain the moving-edges at the reference frame.

The rest of this paper is organized as follows. Section 2 introduces the basic concepts of heat flow. Section 3 discusses anisotropic heat flow for edge map enhancement. Section 4 introduces our novel moving-edge detection method. Section 5 concerns evaluation and experimental results, prior to conclusions.

\section{Basic concepts of heat flow}

In Physics, heat is the form of energy transferred due to the temperature difference within or between bodies. Conduction, convection and radiation are three different modes of heat flow (Holman, 2002). Here, we chose to investigate use of a conduction model, since it is possible and easier to investigate numerical heat conduction problem in the image and in the video domains. The image and video domains are formed by a set of points and these domains are convenient for the finite difference technique.

Conduction is the flow of heat energy from high- to lowtemperature regions due to the presence of a thermal gradient in a body (Holman, 2002). According to Fourier's law of heat conduction, the heat flow rate per unit area is,

$f=-k(\partial T / \partial x)$

where $f$ represents the heat-flow rate, $k$ is positive constant that is called the thermal conductivity of a material, $T$ is the temperature, $x$ is the position, $\partial T / \partial x$ is the temperature gradient and the minus sign indicates that heat flows in the opposite direction to the temperature gradient, satisfying the second principle of thermodynamics.

If we consider a one-dimensional heat conduction system for an element of thickness $d x$ and area $A$ as shown in Fig. 2, by the conservation of energy, the energy conducted in at the left face plus the energy generated within element should be equal to the 


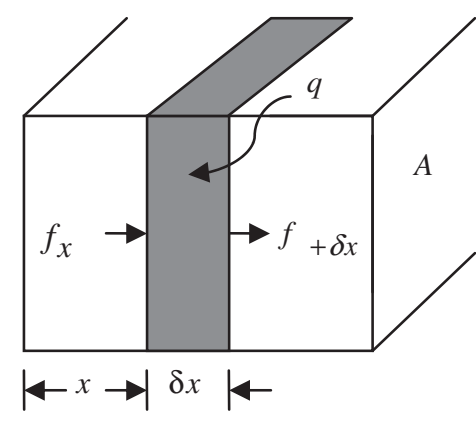

Fig. 2. One-dimensional heat conduction.

change in internal energy plus the energy conducted out at the right face. These energy quantities are represented mathematically as follows:

Energy in at the left face $=f_{x}=-k A(\partial T / \partial x)$

Energy generated within element $=q A \delta x$

Change in internal energy $=\rho c A(\partial T / \partial t) \delta x$

Energy out at the right face $\left.f_{x+\delta x}=-k A(\partial T / \partial x)\right]_{x+\delta x}$

where $q$ is a heat source, which generates energy within element, $c$ is the specific heat of the material and $\rho$ is its density. Combining these relations gives

$-k A \frac{\partial T}{\partial x}+q A \delta x=\rho c A \frac{\partial T}{\partial t} \delta x-A\left[k \frac{\partial T}{\partial x}+\frac{\partial}{\partial x}\left(k \frac{\partial T}{\partial x}\right) \delta x\right]$

The last term in Eq. (6), which is the energy out at the right face of the element, is obtained by the first order Taylor series expansion of Eq. (5). After simplification, we obtain the general heat conduction or diffusion equation,

$\partial T / \partial t=\alpha\left(\partial^{2} T / \partial x^{2}\right)+Q$

where $\alpha=k / \rho c$ is called thermal diffusivity of the material and a larger values of $\alpha$ indicate faster heat diffusion through the material. $Q=q / \rho c$ is the source term that applies internal heating as a function of heat source. It can be uniformly or non-uniformly distributed over material body.

In our application, we consider an image to be a material with property $\rho c=1$ and without internal heat generation (no heat source). These assumptions simplify Eq. (7) into the following form,

$\partial T / \partial t=k\left(\partial^{2} T / \partial x^{2}\right)$

In this case, $\partial T / \partial t$ describes change in internal energy or heat flow per unit area and per unit distance. The two-dimensional heat conduction equation can also be derived by the process explained above and obtained as

$\partial T / \partial t=k\left(\partial^{2} T / \partial x^{2}+\partial^{2} T / \partial y^{2}\right)=k \Delta T$

where $\Delta$ represents the spatial Laplace operator. Assuming that the brightness at a point of a grey level image represents temperature value, the change in brightness with time is related to the rate of heat flow. Here, we note that Eqs. (8) and (9) are obtained by assuming the material has constant conductivity, $k$, which is called uniform conductivity and in this case the heat equation is linear. If the conductivity of a material varies from point to point as a function of position or temperature, the material has non-uniform conductivity and the heat equation is non-linear (Ozisik, 1977). Furthermore, if a material has conductivity which is same in all directions the material medium is called isotropic. Alternatively, a material medium is called anisotropic, which means there exists directional variation of conductivity. Eqs. (8) and (9) are obtained by assuming that material has uniform conductivity and is an isotropic medium. The solution of Eq. (9) provides the temperature distribution over the material body and it depends on time, distance, properties of material, as well as on specified initial and boundary conditions.

Initial conditions specify the temperature distribution in a body, as a function of space, at the origin of the time coordinate $(t=0)$. Initial conditions are represented as follows:

$T(\boldsymbol{x}, t=0)=F(\boldsymbol{x})$,

where $\boldsymbol{x}=(x, y)$ is the space vector for the two-dimensional case and $F(\boldsymbol{x})$ is the function that specifies the initial temperature inside the body.

Boundary conditions specify the temperature or the heat flow at the boundaries of the body. There are three general types of boundary conditions: Dirichlet, Neuman and Robin. Here, we consider the Dirichlet and Neuman conditions, which are used in our algorithm. In the Dirichlet condition, temperature is specified along the boundary layer. It can be a function of space and time, or constant. The Dirichlet condition is represented as follows:

$T(\boldsymbol{x}, t)=\Phi(\boldsymbol{x}, t)$

where $\Phi(\boldsymbol{x}, t)$ is the function that specifies the temperature at the boundary layer.

In the Neuman condition, the normal derivative of temperature, heat flow, is defined at the boundary surface, and it can be a function of space and time, or constant. The Neuman condition is given in the form

$\frac{\partial T(\boldsymbol{x}, t)}{\partial n}=\Phi(\boldsymbol{x}, t)$

where $\Phi(\boldsymbol{x}, t)$ is the function that specifies the normal derivative of temperature, $\partial T(\boldsymbol{x}, t) / \partial n$, at the boundary layer.

The numerical heat conduction problem can be investigated in the image and video domains, since these domains are formed by a set of points, as well as since they are convenient for the finite difference technique. In our algorithm, we use the finite difference technique for the solution of the heat conduction problems. We do not consider the finite element methods, since our aim is to use the heat analogy for feature extraction. As such, the finite element methods would become rather complex for this aim.

\section{Anisotropic heat diffusion and edge enhancement}

Perona and Malik (1990) proposed anisotropic diffusion for selective image smoothing that avoids blurring and localization problems of the edges. The anisotropic heat diffusion problem is given below:

$\frac{\partial \boldsymbol{I}}{\partial t}=\operatorname{div}(k(\boldsymbol{x}, t) \nabla \mathbf{I}(\boldsymbol{x}, t))$

$\boldsymbol{I}(\boldsymbol{x}, t=0)=\boldsymbol{F}(\boldsymbol{x}), \quad$ initial condition

$\partial \boldsymbol{I}(\boldsymbol{x}, t) / \partial n=0, \quad$ boundary condition

where div represents divergence operator, $\nabla$ is a spatial gradient operator, $\boldsymbol{I}(\boldsymbol{x}, t)$ is the grey level image that is a function of position $\boldsymbol{x}=(x, y)$ and time $t, k(\boldsymbol{x}, t) \in[0,1]$ is the conductivity coefficient and can be chosen to be a function $k(\boldsymbol{x}, t)=g(|\nabla \mathbf{I}(\boldsymbol{x}, t)|)$, which depends on directional derivative of $\boldsymbol{I}(\boldsymbol{x}, t)$ at each position and time. The original image, $\boldsymbol{F}(\boldsymbol{x})$, indicates the initial condition. The boundary of the image is insulated, which is represented with a homogeneous Neuman condition. In this boundary condition, there is no heat flow in or out of the image from the boundary. The anisotropic diffusion equation is derived when the conductivity of a material (image) has 


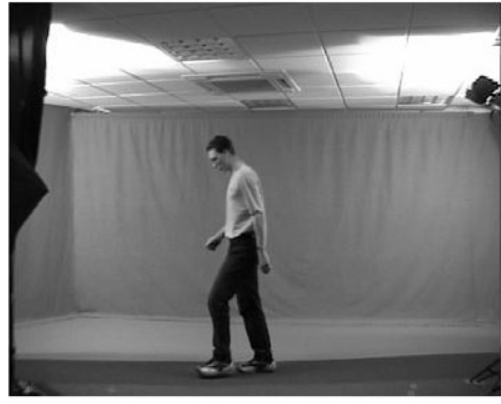

(a) Grey-scale image

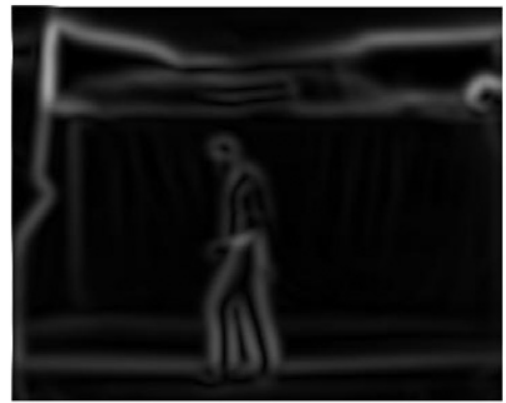

(c) Sobel edge map after isotropic diff.

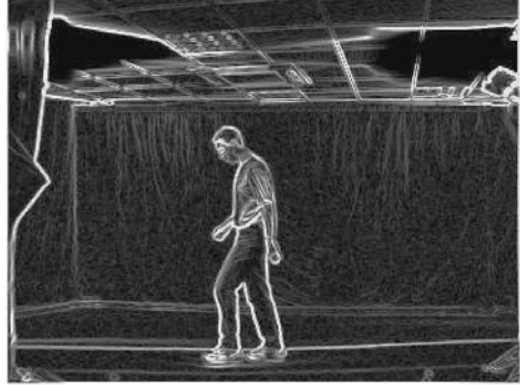

(b) Original Sobel edge map

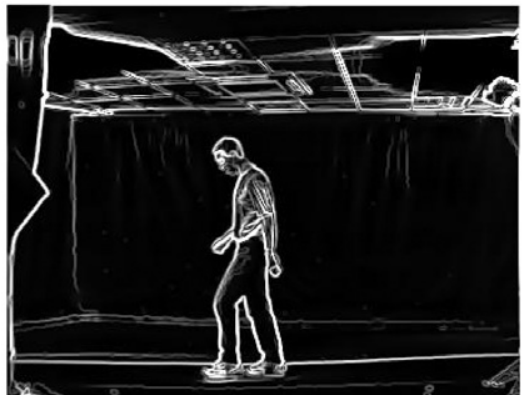

(d) Sobel edge map after anisotropic diff.

Fig. 3. Difference between isotropic and anisotropic diffusion.

directional variations at each position and time. If the thermal conductivity is constant in all direction at each position and time, Eq. (13) can be simplified to Eq. (9) in 2-D.

Different functions are used for $g(|\nabla \mathbf{I}|)$ depending on the chosen aim (image segmentation, edge detection, etc) as described in Perona and Malik (1990). In our application, an exponential type is used, (see Eq. (14)), which prefers high-contrast edges to lowcontrast ones.

$g(\nabla \boldsymbol{I})=e^{-(|\nabla \boldsymbol{I}| / R)^{2}}$

$R$ determines the rate of decay of the exponential function, and thus the rate of smoothing. Note that, if $g(|\nabla \boldsymbol{I}|)$ is constant (at all image locations), this leads to isotropic heat diffusion. In Fig. 3, we illustrate the difference between isotropic and anisotropic diffusion operations. Fig. 3(a) is a grey-scale image and Fig. 3(b) is its Sobel edge map without any diffusion. Fig. 3(c) is the Sobel edge map after isotropic diffusion, which causes loss of edge information. On the other hand, Fig. 3(d) is the Sobel edge map of the anisotropic diffused image with the conductivity function given by Eq. (14) and it can easily be observed that high contrast edges are enhanced while removing-edges due to noise, and thus important detail is preserved. Note that we choose to use the Sobel operator as a finite difference edge finder. Because, it is one of the best finite difference edge detection operator (Nixon and Aguado, 2007) and it performs better than other contemporaneous edge detection operators, such as Prewitt operator.

\section{Isotropic heat flow in temporal domain}

Here, we introduce a novel moving-edge detection technique. Assume that we have three or more consecutive frames. Once the enhanced Sobel edge maps are obtained in space, the isotropic and linear heat equation with the proposed initial and boundary conditions is applied in the temporal domain to calculate the total amount of heat flow. Assuming we have $L$ consecutive frames, the discrete formulation of the proposed heat conduction problem is given below:
$\boldsymbol{E}_{n}^{t}=\boldsymbol{E}_{n}^{t-1}+k \Delta \boldsymbol{E}_{n}^{t-1}=\boldsymbol{E}_{n}^{t-1}+k\left(\boldsymbol{E}_{n+1}^{t-1}+\boldsymbol{E}_{n-1}^{t-1}-2 \boldsymbol{E}_{n}^{t-1}\right)$

$\boldsymbol{E}_{n}^{t=0}=\boldsymbol{F}_{n} \quad$ initial condition

$\boldsymbol{E}_{n=0}^{t}=\boldsymbol{E}_{0}^{t=0}, \quad \boldsymbol{E}_{n=L-1}^{t}=\boldsymbol{E}_{L-1}^{t=0} \quad$ boundary conditions

where $\boldsymbol{E}_{n}^{t-1}, \boldsymbol{E}_{n-1}^{t-1}$ and $\boldsymbol{E}_{n+1}^{t-1}$ are Sobel edge mapped images respectively for the reference frame $(n)$, the previous frame $(n-1)$ and the next frame $(n+1)$ at iteration $t, \Delta$ is Laplacian operator, $k$ is thermal conductivity and $0 \leqslant k \leqslant 0.5$ for the numerical scheme to be stable in the one-dimensional case (Holman, 2002). The original image sequences, $\boldsymbol{F}_{n}$, indicates the initial condition and the proposed boundary condition is Dirichlet with constant temperature. In this boundary condition, the boundary frames always remain at the initial values and there will be heat flow in and out of the image sequences. Since we assume there are moving objects in the consecutive images, moving-edge positions in any interior frame are expected to have higher brightness than the same positions at the boundary frames. As a result of this, moving-edges diffuse out from the reference frame to the neighbour frames and then out of image sequences from the boundary frames.

Diffusion (the iteration) ceases, when we reach the steady-state solution in the system, when there is no more heat flow. The total amount of heat flow, for each frame, is calculated as follows. Assume that the initial scale is 0 (zero) and final scale is $t$, then Eq. (15) can be described as:

$\boldsymbol{E}_{n}^{t}=\boldsymbol{E}_{n}^{0}+k \sum_{i=0}^{t-1} \Delta \boldsymbol{E}_{n}^{i}$

Then, the total amount of heat flow from the initial state to the steady-state is

$\left|\boldsymbol{E}_{n}^{t}-\boldsymbol{E}_{n}^{0}\right|=k \sum_{i=0}^{t-1}\left|\Delta \boldsymbol{E}_{n}^{i}\right|$

However, this gives us total heat in (+) and heat out (-) together during diffusion. We are interested in total heat flow out (HFO) from the reference frame, $\boldsymbol{E}_{n}$, which gives us the moving-edge map. This is obtained as, 
HFO $=k \sum_{i=0}^{t-1}\left|\Delta \boldsymbol{E}_{n}^{i}\right|, \quad \forall \Delta \boldsymbol{E}_{n}^{i}<0$

Fig. 4 illustrates the process for three consecutive frames, where Fig. $4 a-c$ show successive images of a walking human subject. First, anisotropic diffusion is applied in space with parameters values $\lambda=0.2, R=4$ and for 50 iterations. Fig. $4 \mathrm{~d}-\mathrm{f}$ show enhanced Sobel edge maps of these frames after anisotropic diffusion. Then, isotropic diffusion is applied in temporal domain to calculate total amount of heat flow. This diffusion continues until we observe steady-state with $\alpha=0.45$, which needs 10 iterations in this experiment. Fig. 4(g) shows total amount of heat flow in the reference

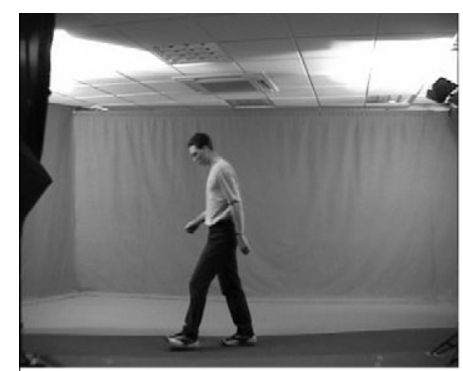

(a) Frame $_{n+1}$

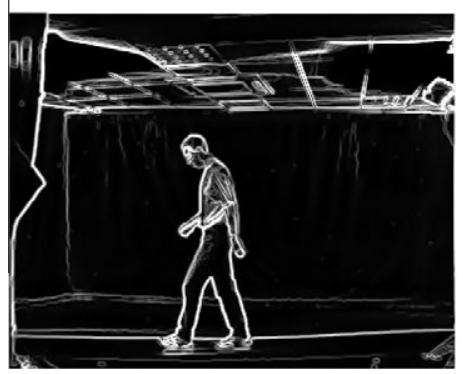

(d) $\mathbf{E}_{n+1}$

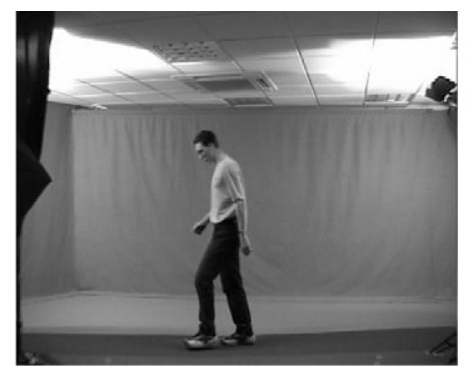

(b) Frame $_{n}$

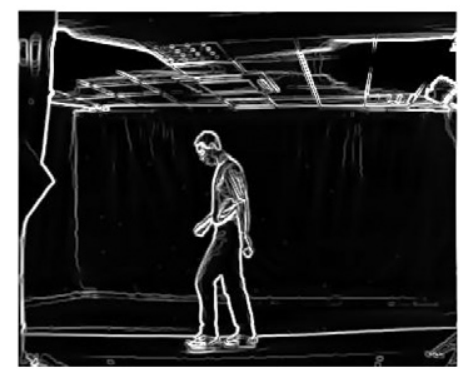

(e) $\mathbf{E}_{n}$

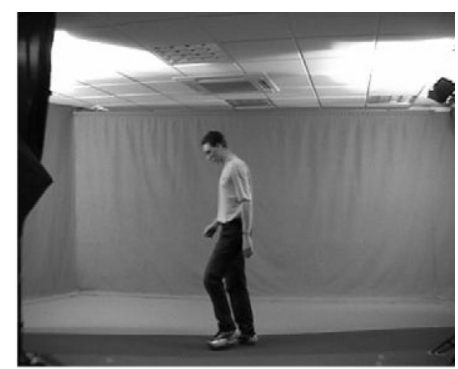

(c) Frame $_{n-1}$

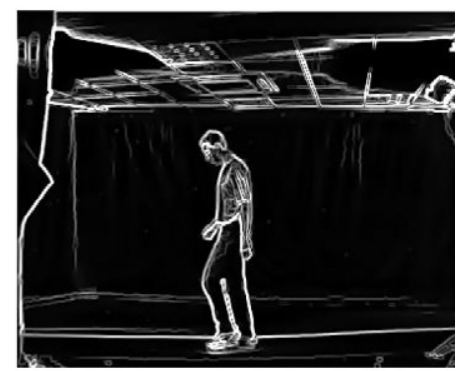

(f) $\mathbf{E}_{n-1}$

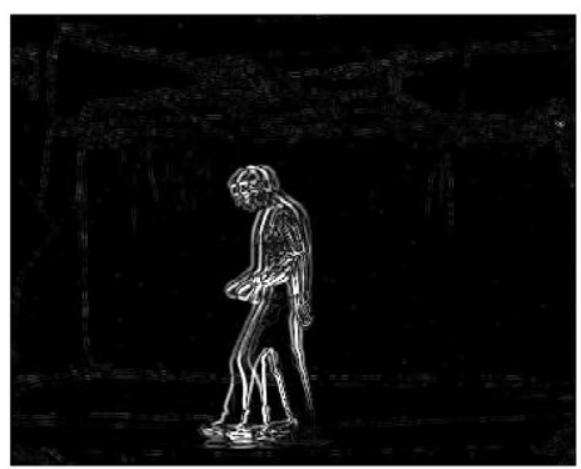

(g) Total heat flow

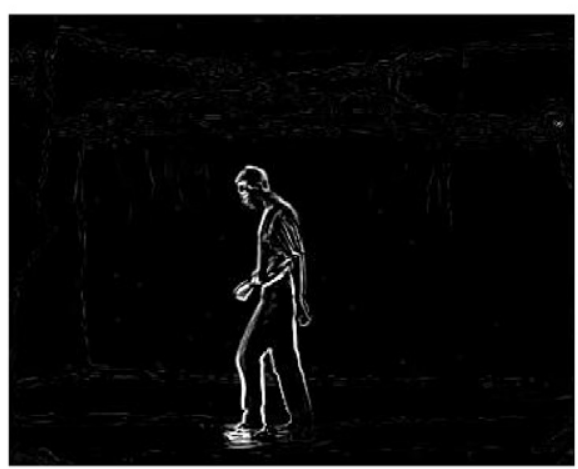

(h) Heat flow out (HFO)

Fig. 4. Moving-edge map extraction.

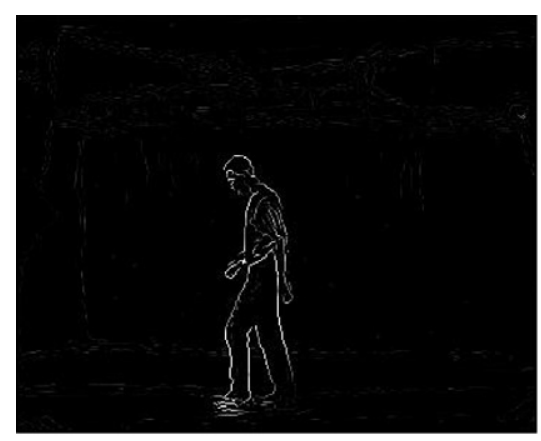

(a) Non-maxima suppressed image

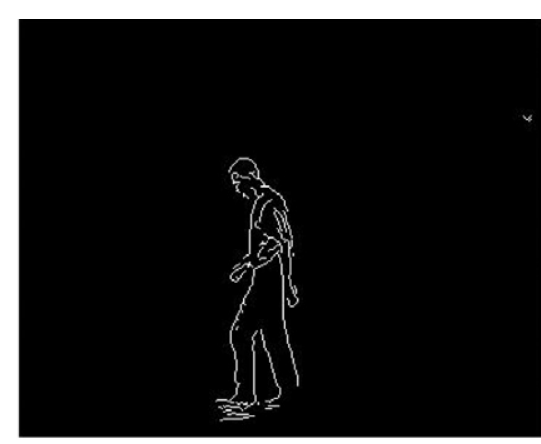

(b) Hysteresis thresholded image

Fig. 5. Binary moving-edge observation. 
frame, $\boldsymbol{E}_{n}$, and Fig. $4(\mathrm{~h})$ is total heat flow out from $\boldsymbol{E}_{n}$, which gives the moving-edge map. Only the moving-edges of the human subject and some slight shadows remain, whilst largely removing the edges introduced by the static background.

The overall process is completed by non-maxima suppression (thinning) and hysteresis thresholding to observe the binary moving-edges. The non-maxima suppressed and hysteresis thresholded images are shown respectively in Fig. 5(a) and (b).

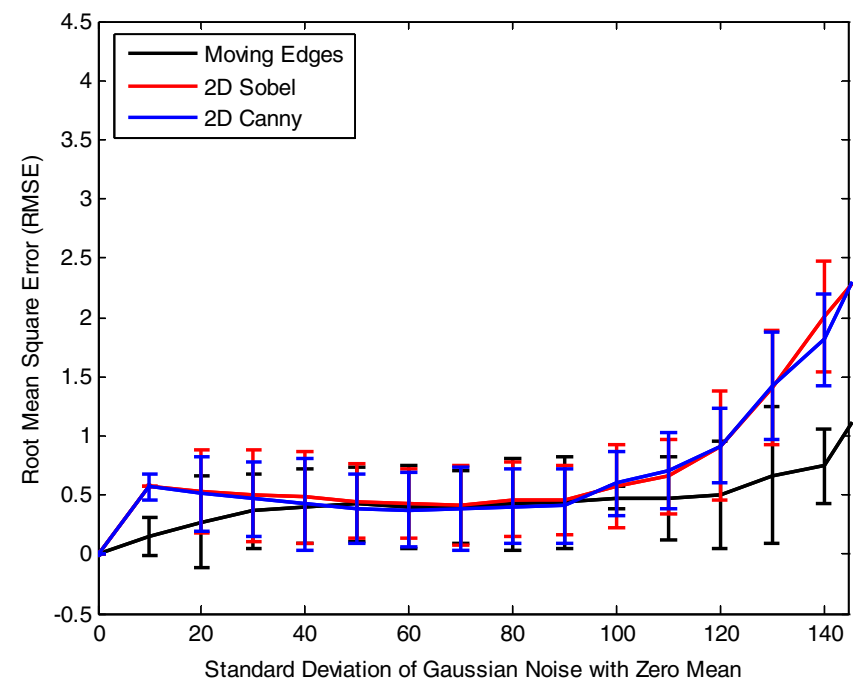

Fig. 6. Performances of moving-edges, 2-D Sobel and 2-D Canny with respect to normally distributed noise trials. RMSE is plotted with mean and standard deviation values using error bars.

\section{Evaluation and experimental results}

Performance evaluation is employed by comparing movingedge detection with 2-D Canny edge detection and 2-D Sobel edge detection. Evaluation is performed using a white circle moving on a black background with different levels of normally distributed noise $N_{d}\left(\mu, \sigma^{2}\right)$. Binary edge images are computed at the reference frame by moving edges, 2-D Canny and 2-D Sobel algorithms, separately. The Hough Transform (HT) is applied to the binary edge images to extract the circle's centre parameters. A root mean square error (RMSE) is then employed to quantify the performance of each algorithm.

$\operatorname{RMSE}=\sqrt{\left(\left(e_{x}-c_{x}\right)^{2}+\left(e_{y}-c_{y}\right)^{2}\right) / 2}$

where $\left(e_{x}, e_{y}\right)$ are the extracted circle centre parameters and $\left(c_{x}, c_{y}\right)$ are the actual circle centre parameters. The quantity of noise is considered in terms of standard deviation $\sigma$, with zero mean.

Note that (moving) edge detection is a low-level feature extraction (Nixon and Aguado, 2007), which do not give shape information of the objects. Naturally, edges can be used within high-level feature extraction to find shapes. Hough Transform (HT) is a highlevel feature extraction that uses detected edges of the object to estimate the shape. In our evaluation, the HT extracts circle centre position from the edges and the RMSE computes dislocation of the circle centre position (that means dislocation of the edges). Dislocation in the circle centre position occurs since the detected edges are affected by the noise in the image. The proposed evaluation can give an idea about how much the proposed method, 2-D Canny and 2-D Sobel operators can resist to the noise and can give accurate edges with respect to increasing noise.
Original
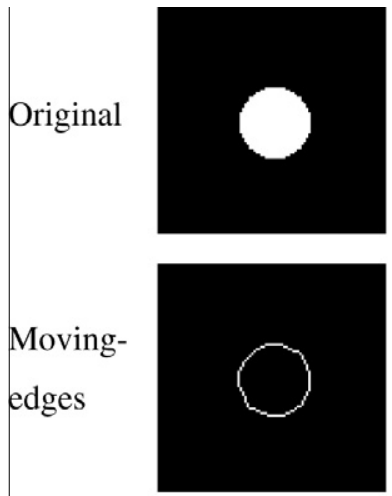

2-D

Sobel

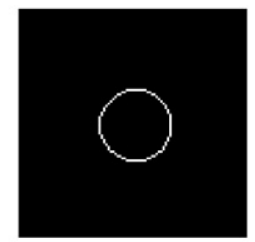

2-D

Canny

565

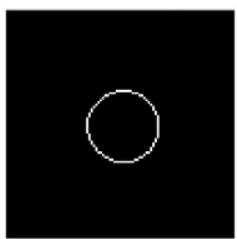

(a) $\sigma=0$
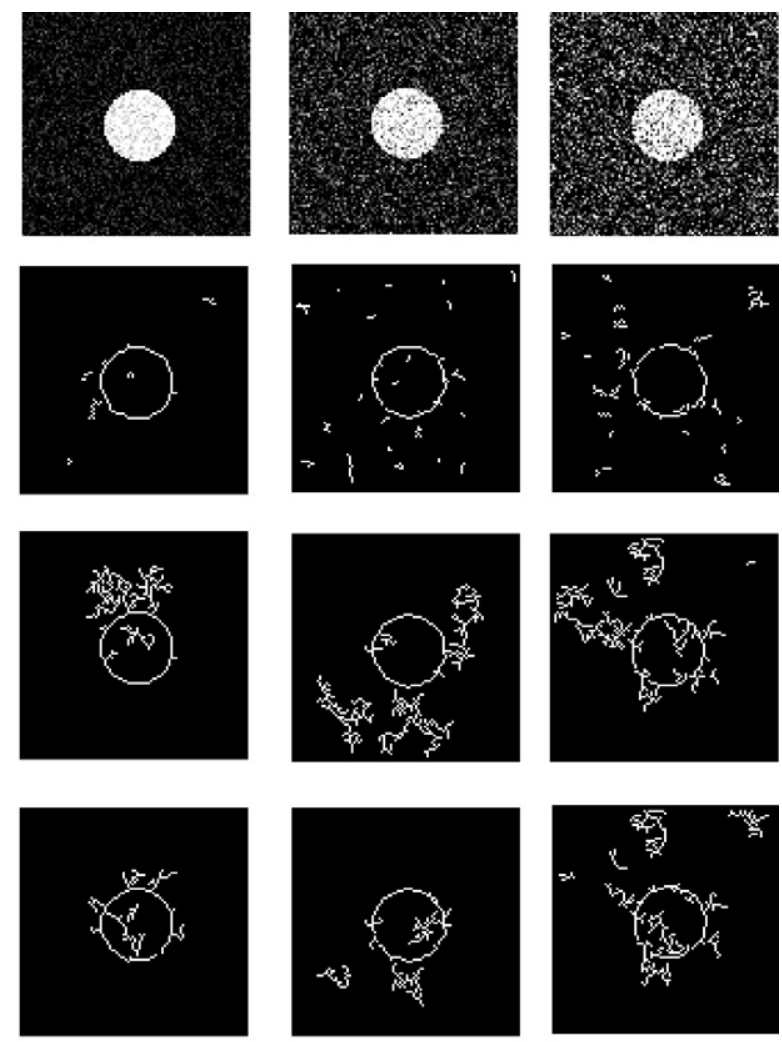

(c) $\sigma=80$

(d) $\sigma=120$

Fig. 7. Results for moving-edges (second row), 2-D Sobel (third row) and 2-D Canny (fourth row) with respect to increasing Gaussian noise. 


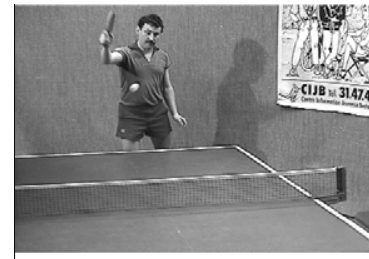

frame $_{1}$

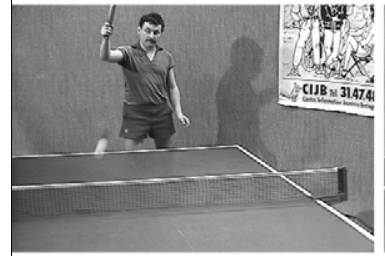

frame $_{5}$

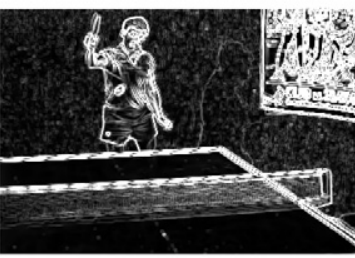

$\mathbf{E}_{l}$

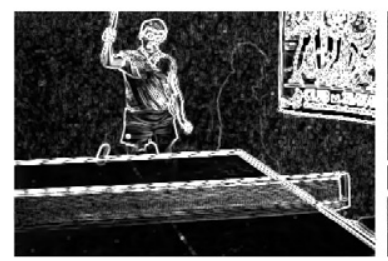

$\mathbf{E}_{5}$

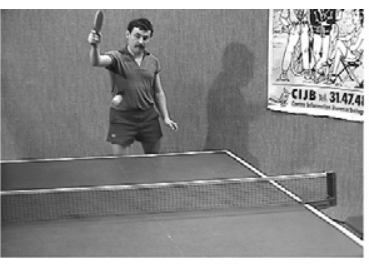

frame $_{2}$

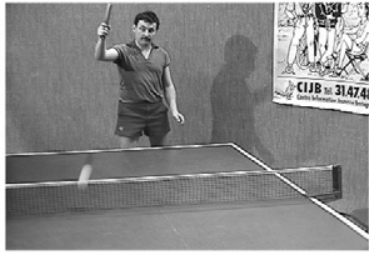

frame $_{6}$

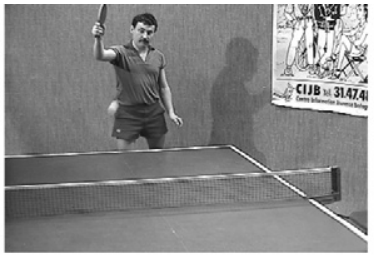

frame $_{3}$

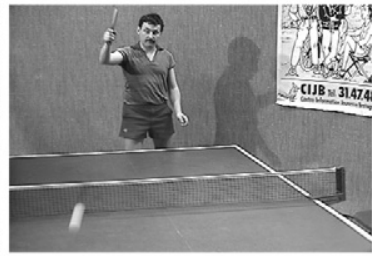

frame $_{7}$

(a) Input frames

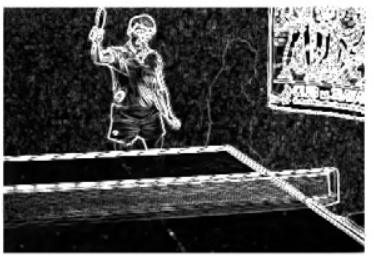

$\mathbf{E}_{2}$

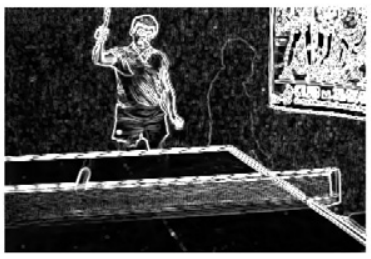

$\mathbf{E}_{6}$

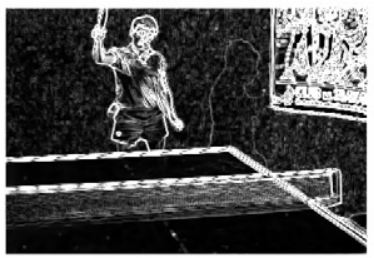

$\mathbf{E}_{3}$

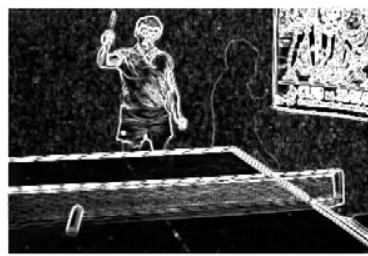

$\mathbf{E}_{7}$

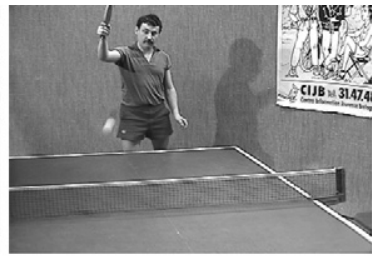

frame $_{4}$

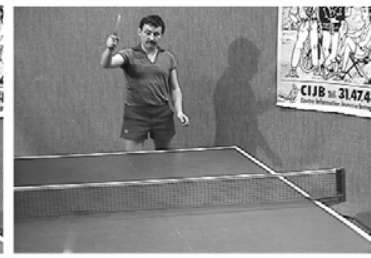

frame $_{8}$

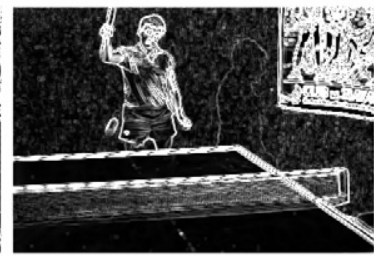

$\mathbf{E}_{4}$

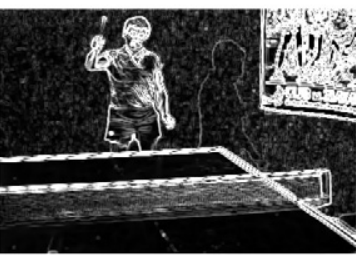

$\mathbf{E}_{8}$

(b) Enhanced Sobel edge maps after anisotropic diffusion

Fig. 8. Moving-edge detection in the eight consecutive frames.

The Canny edge detection is perhaps the most popular edge detection technique at present. The first requirement, for the Canny edge detection, is to reduce the response to noise with Gaussian filtering. Then, a finite difference edge finder is applied to compute the gradient magnitude. The optimal finite difference edge finder for the Canny edge detection algorithm is given in (Fleck, 1992), which we use in our evaluation. Then non-maxima suppression (peak detection) is applied to the gradient magnitude image that retains only those points at the top of the ridge, whilst suppressing others. Finally, hysteresis thresholding is used, which involves two thresholds, to obtain binary edges. In our evaluation, we omit the Gaussian filtering stage (pre-processing), since we aim to observe performance of the algorithm with respect to increasing noise. The thresholds for the 2-D Canny, to obtain the binary image, are determined by a root mean square (RMS) estimate of the noise. In this process, the non-maxima suppressed image is thresholded by scaled mean value of the gradient magnitude image that is proportional to signal to noise ratio (SNR). The upper and the lower thresholds are determined as given below:

$T_{H}=c_{T} \times \frac{1}{M N} \sum_{x=1}^{M} \sum_{y=1}^{N} \boldsymbol{c}_{x, y}, \quad T_{L}=T_{H} / 4$ where $\boldsymbol{C}$ is the gradient magnitude reference frame of size $M \times N . c_{T}$ is a scale factor for threshold selection, which is a positive constant. Higher values of $c_{T}$ cause higher values for the thresholds that can filter out noise better. However, the threshold values must be smaller than the maximum intensity values of the image, which we are thresholding. In this evaluation, the optimum value for $c_{T}$ is 3 that is determined experimentally. The ratio between high, $T_{H}$, and low, $T_{L}$, thresholds is 4 .

The 2-D Sobel operator was the most popular edge detection operator until the development of edge detection techniques with a theoretical basis, such as Canny edge detection (Nixon and Aguado, 2007). It proved popular because it gave, overall, a better performance than other contemporaneous edge detection operators, such as Prewitt operator. In our evaluation, there is no pre-processing or smoothing stage before the Sobel operation since we aim to observe performance of the operator with respect to increasing noise. After computing the gradient magnitude with the Sobel operator, non-maxima suppression and Hysteresis thresholding is used to obtain thin and binary edges. Note that this algorithm is similar to the 2-D Canny algorithm. However, the finite difference edge finding is achieved with the 2-D Sobel operator. The thresholds for the 2-D Sobel, to obtain the binary image, are also 


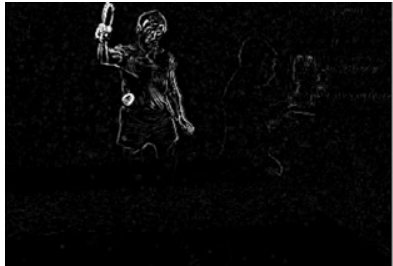

$\mathrm{HFO}_{2}$

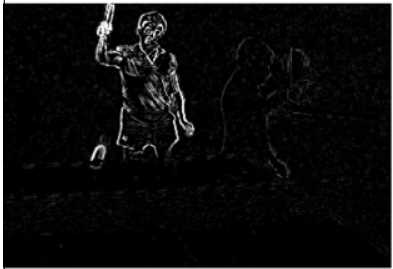

HFO $_{5}$

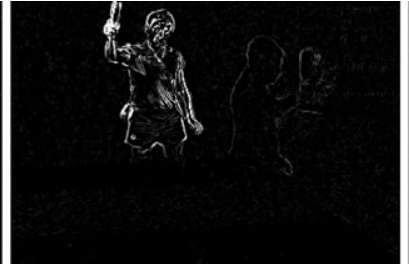

$\mathrm{HFO}_{3}$

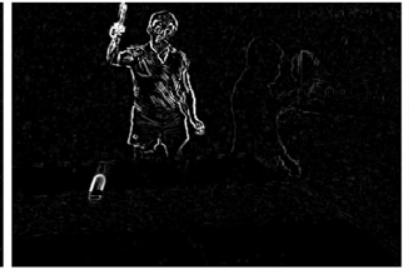

$\mathrm{HFO}_{6}$

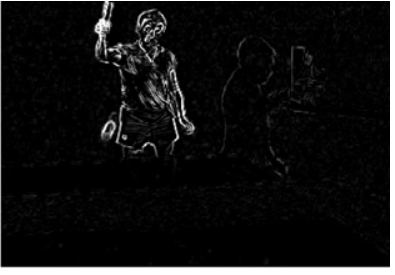

$\mathrm{HFO}_{4}$

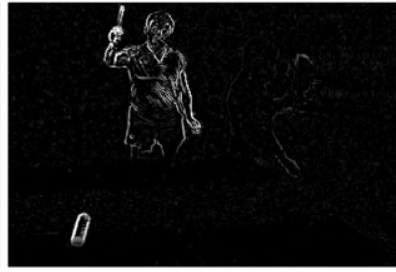

$\mathrm{HFO}_{7}$

(c) Heat flow out (-) from reference frames until the steady-state

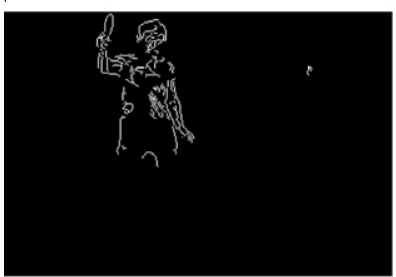

Binary_Edge $e_{2}$

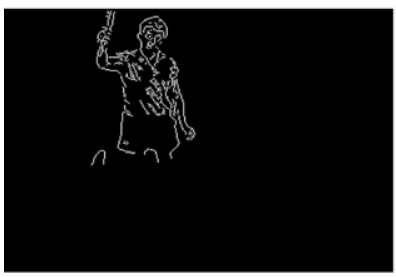

Binary_Edge $e_{5}$

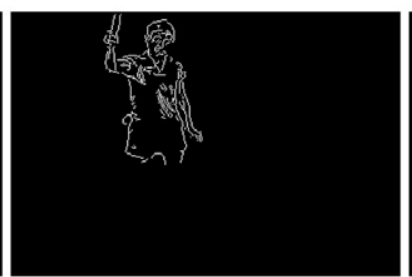

Binary_Edge

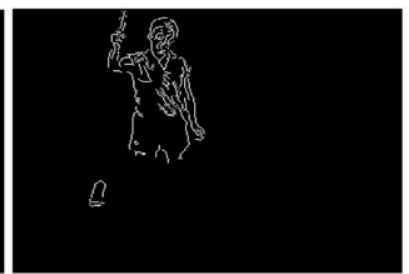

Binary_Edge $_{6}$

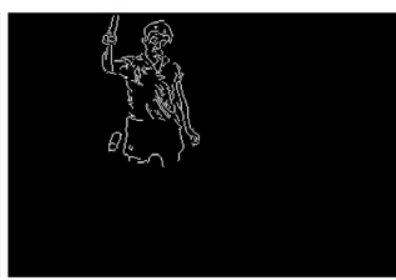

Binary_Edge $e_{4}$

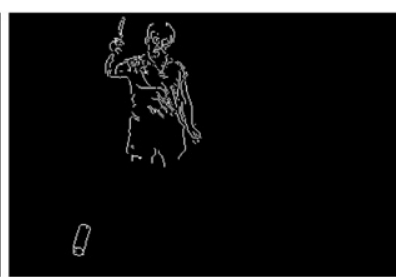

Binary_Edge $_{7}$

(d) Binary moving-edges after non-maxima suppression and hysteresis thresholding

Fig. 8 (continued)

determined by a root mean square (RMS) estimate of the noise. The non-maxima suppressed image is thresholded by scaled mean value of the gradient magnitude image that is proportional to signal to noise ratio (SNR). The upper and the lower thresholds are determined as given below:

$T_{H}=S_{T} \times \frac{1}{M N} \sum_{x=1}^{M} \sum_{y=1}^{N} \boldsymbol{S}_{x, y}, \quad T_{L}=T_{H} / 4$

where $\boldsymbol{S}$ is the gradient magnitude reference frame of size $M \times N$ and $s_{T}$ is a scale factor for threshold selection, which is a positive constant with value 3 in our evaluation. The value of $s_{T}$ is determined experimentally same as we did in 2-D Canny algorithm. The value of $s_{T}$ is optimal, which provides thresholds not higher than maximum intensity values of the image and which provides better thesholds to filter out noise. The ratio between high, $T_{H}$, and low, $T_{L}$, thresholds is 4 .

In the moving-edge detection algorithm, the anisotropic heat diffusion in the spatial domain (the pre-processing stage) is also omitted to observe the response of the proposed operator to the noise. Hysteresis thresholding is applied to the non-maxima suppressed HFO image at the reference frame. The thresholds of the moving-edge detection algorithm are based on mean value of the HFO image,

$T_{H}=h_{T} \times \frac{1}{M N} \sum_{x=1}^{M} \sum_{y=1}^{N} \mathbf{H F O}_{x y}, \quad T_{L}=T_{H} / 4$

where $h_{T}$ is a scale factor for threshold selection with value 11 and the ratio between the high, $T_{H}$, and the low, $T_{L}$, thresholds is again 4 . Here, the scale factor, $h_{T}$, is also determined experimentally and it is optimal to prevent the threshold values become higher than maximum intensity values of the HFO image and to achieve better thresholding to filter noise out. Fig. 6 shows performance of moving-edge detection, 2-D Canny and 2-D Sobel algorithms. The graphs include error bars representing the mean and standard deviation obtained over five applications of the algorithms. In each application, the obtained graphs are smoothed by applying local averaging to the data points in six nearest neighbourhoods. It is observed that 2-D Canny and 2-D Sobel have similar performances. RMSE of the 2-D Canny and RMSE of the 2-D Sobel increase until $\sigma \cong 10$. Then, they are stable between $\sigma \cong 10$ and $\sigma \cong 90$. After $\sigma \cong 90$, we observe increase in RMSE of 2-D Canny and RMSE of 2-D Sobel. Moving-edges has better performance than 2-D Canny and 2-D Sobel until $\sigma \cong 40$, since 

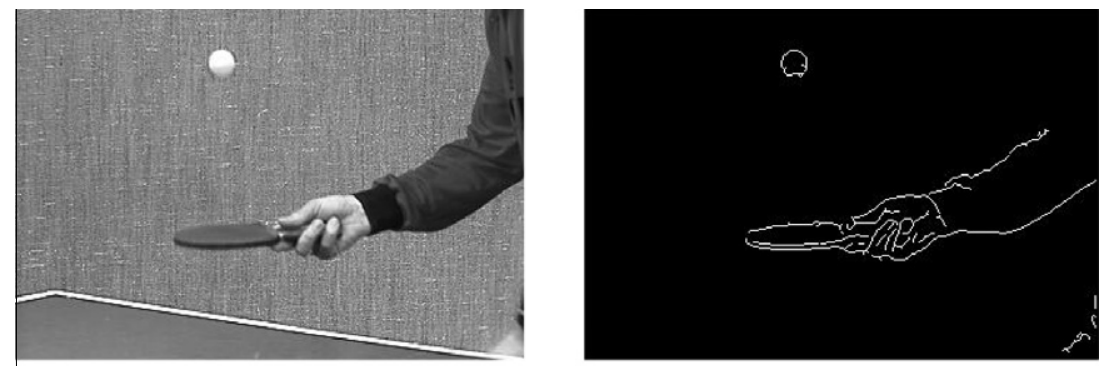

(a) Table tennis (indoor) image
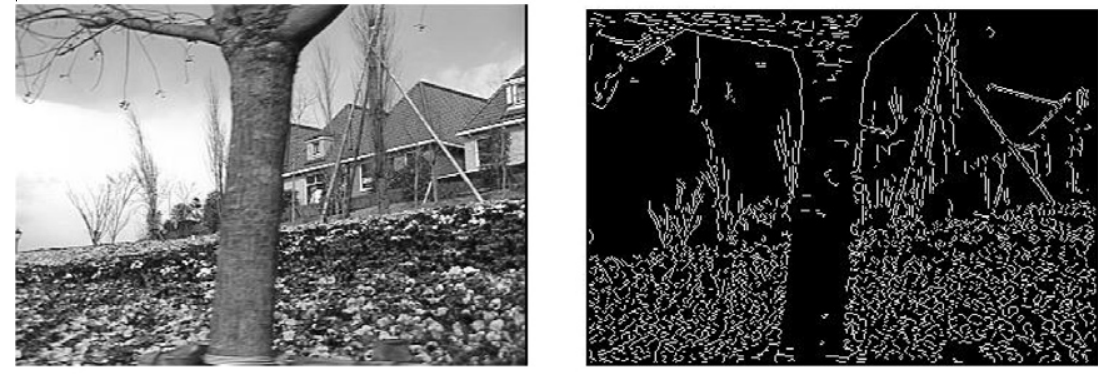

(b) Flower garden (outdoor) image, where the camera is in motion.

Fig. 9. Some of the simulation results for new operator on indoor and outdoor images.

the RMSE of the moving-edges increases slower than other algorithms until $\sigma \cong 40$. Moving-edges has similar performance with 2-D Canny and 2-D Sobel between $\sigma \cong 40$ and $\sigma \cong 90$, where the RMSE of the moving-edges is stable in that interval. After $\sigma \cong 90$ at higher noise levels, it is observed that moving edge detection technique has better performance than 2-D Canny and 2-D Sobel, which appears due to the averaging inherent in the new operator. Fig. 7 shows some of the results for moving-edges (second row), 2-D Sobel (third row) and 2-D Canny (fourth row). To visual inspection, the input images in Fig. 7(c) and (d), are very noisy indeed.

Simulation results also show that our algorithm can detect moving-edges in image sequences, as shown in Fig. 8. Fig. 8(a) has eight consecutive frames and shows a human subject, playing table tennis. Fig. 8(b) shows Sobel edge maps, E, after the anisotropic diffusion in space. The selected parameters for anisotropic diffusion: $\lambda=0.2, R=4$ and the number of iterations is 20 . It is observed that region boundaries are sharpened after anisotropic diffusion. Although almost all noise has been removed on the table, the wall behind the player still appears noisy. The reason for this is the textured structure of the wall and there are strong edges as well. The textured structure of the wall can bee seen in Fig. 9(a), since this image is enlarged and also belongs to the same table tennis sequence. Fig. 8(c) is the total amount of heat flow out (HFO) from each frame, after isotropic diffusion in the temporal domain. This diffusion needs 215 iterations with $\alpha=0.45$ to achieve steady-state. Here, the first and eighth frames are omitted since they are the boundary frames and their edge map values are constant, during the diffusion, to achieve the given Dirichlet boundary conditions. It is observed that almost all edges introduced by the static background and the noise arising from the textured wall is removed, while retaining the moving-edges of the human subject and the tennis ball. There are also slight edges of the human's shadow in the HFO images. Fig. 8(d) shows the binary moving-edges at each reference frame after applying non-maxima suppression and hysteresis thresholding. It is seen that movingedges were indeed detected. This experiment also shows that the proposed algorithm is also able to detect moving-edges, which belong to the different moving objects in the scene (the tennis player and the tennis ball).
Fig. 9 also shows some simulation results. Fig. 9(a) shows the reference frame from table tennis (indoor) sequence and movingedges of the arm and of the ball were detected. We should note that the upper part of the arm and the table are static and this is why they were not detected. Fig. 9(b) is a reference frame from the flower garden (outdoor) sequence, where the camera is in motion. It is seen again that most of the moving-edges were detected. Some edges, such as part of the house roofs, were not detected, since the camera was moving along those edges.

\section{Conclusions}

We have presented a novel low level moving-feature extraction technique based on using the heat flow analogy. Firstly, high quality Sobel edge maps are obtained based on anisotropic heat diffusion, in space. The conductivity function is the key point in this stage; we have chosen an exponential function which enhances high contrast edges and removes edges due to noise. In the next stage, isotropic and linear heat diffusion is applied in the temporal domain to determine the moving-edge map in the reference frame. To do this, the total amount of heat flow is calculated and then separated into the heat in and heat out parts, where the heat out is the moving-edge map. Finally, non-maxima suppression and hysteresis thresholding is applied to obtain binary moving-edges. Evaluation indicates that this technique is better than edge detection techniques, which is based on 2-D Canny and 2-D Sobel operators, without anisotropic heat diffusion in space. This result appears to be due to the averaging inherent in the new operator. Results also show that this technique can detect moving-edges in image sequences, without using the background detection.

\section{References}

Ahn, Y., Ahn, K., Chae, O., 2004. Detection of moving objects edges to implement home security system in a wireless environment. In: Internat. Conf. on Computer Science and Its Applications, LNCS, vol. 3043, pp. 1044-1051.

Caselles, V., Catte, F., Coll, T., Dibos, F., 1993. A geometric model for active contours. Numer. Math. 66, 1-31. 
Dewan, M.A.A., Hossain, M.J., Chae, O., 2007. Reference independent moving object detection an edge segment based approach. In: Internat. Conf. on Knowledgebased and Intelligent Information and Engineering System, LNAI, vol. 4692, pp. 501-509.

Direkoğlu, C., Nixon, M.S., 2006. Low level moving-feature extraction via heat flow analogy. In: Internat. Symp. on Visual Computing, LNCS, vol. 4291, pp. 243-252.

Direkoğlu, C., Nixon, M.S., 2007. Shape extraction via heat flow analogy. In: Internat. Conf. on Advanced Concepts for Intelligent Vision, LNCS, vol. 4678, pp. 553-564.

Fleck, M.J., 1992. Some defects in finite-difference edge finders. IEEE Trans. Pattern Anal. Machine Intell. 14 (3), 337-345.

Holman, J.P., 2002. Heat Transfer, nineth ed. McGraw-Hill.

Hwang, T., Clark, J.J., 1990a. On local detection of moving edges. In: Proc. 10th Internat. Conf. on Pattern Recognition, vol. 1, pp. 180-184.

Hwang, T., Clark, J.J., 1990b. A spatio-temporal generalization of Canny's edge detector. In: Proc. 10th Internat. Conf. on Pattern Recognition, vol. 1, pp. 314318.

Ji, X., Feng, J., 2004. A new approach to thinning based on time-reversed heat conduction model. In: IEEE Internat. Conf. on Image Process., vol. 1, pp. 653656.

Kim, S.H., 2005. A novel approach to moving edge detection using cross entropy. In: Internat. Conf. on Graphics, Vision and Image Process., pp. 21-24.

Kim, C., Hwang, J.N., 2002. Fast and automatic video object segmentation and tracking for content-based applications. IEEE Trans. Circuits Systems Video Technol. 12 (2), 122-129.
Kimia, B.B., Siddiqi, K., 1994. Geometric heat equation and nonlinear diffusion of shapes and images. In: IEEE Internat. Conf. on Computer Vision and Pattern Recognition, pp. 113-120.

Makrogiannis, S.K., Bourbakis, N.G., 2004. Motion analysis with application to assistive vision technology. In: IEEE Internat. Conf. on Tools with Artificial Intell., pp. 344-352.

Malladi, R., Sethian, J.A., Vemuri, B.C., 1995. Shape modeling with front propagation: A level set approach. IEEE Trans. Pattern Anal. Machine Intell. 17 (2), 158-175.

Manay, S., Yezzi, A., 2003. Anti-geometric diffusion for adaptive thresholding and fast segmentation. IEEE Trans. Image Process. 12 (11), 1310-1323.

Myerscough, P.J., Nixon, M.S., 2004. Temporal phase congruency. In: IEEE Southwest Symposium on Image Analysis and Interpretation, pp. 76-79.

Nixon, M.S., Aguado, A., 2007. Feature Extraction and Image Processing, second ed. Elsevier.

Nixon, M.S., Liu, X.U., Direkoğlu, C., Hurley, D.J., 2009. On using physical analogies for feature and shape extraction in computer vision. Comput. J.

Osher, S., Sethian, J.A., 1988. Fronts propagating with curvature-dependent speed: Algorithms based on hamilton-jacobi formulations. J. Comput. Phys. 79 (1), $12-$ 49.

Ozisik, M.N., 1977. Basic Heat Transfer. McGraw-Hill.

Perona, P., Malik, J., 1990. Scale-space and edge detection using anisotropic diffusion. IEEE Trans. Pattern Anal. Machine Intell. 22 (8), 629-639.

Zhang, X.Y., Zhao, R.C., 2006. Automatic video object segmentation using wavelet transform and moving edge detection. In: IEEE Internat. Conf. on Machine Learning and Cybernet., pp. 1174-1177. 